\title{
Metformin Intoxication: A Case of a Combination of Severe Hypoglycaemia and Severe Lactic Acidosis (LA)
}

\author{
Dinette Agterhuis ${ }^{1}$, Hanneke Freling1, Margreet Henstra², \\ Thiemo F. Veneman ${ }^{1,3^{*}}$ \\ ${ }^{1}$ Department of Intensive Care Medicine, Ziekenhuisgroep Twente, Almelo, The Netherlands \\ ${ }^{2}$ Department of Pharmacology, Ziekenhuisgroep Twente, Almelo, The Netherlands \\ ${ }^{3}$ Department of Internal Medicine, Ziekenhuisgroep Twente, Almelo, The Netherlands \\ Email: ${ }^{*}$ tfveneman@hetnet.nl
}

Received 23 February 2015; accepted 11 May 2015; published 14 May 2015

Copyright (C) 2015 by authors and Scientific Research Publishing Inc.

This work is licensed under the Creative Commons Attribution International License (CC BY). http://creativecommons.org/licenses/by/4.0/

cc) (i) Open Access

\begin{abstract}
Metformin is a worldwide used and effective antihyperglycemic agent. It is generally well tolerated but contra-indicated in severe renal dysfunction. Metformin does not cause hypoglycaemia, however this case describes hypoglycaemia after an overdose. Another well-known, but rare and potentially life-threatening complication of metformin is the occurrence of LA, the so-called metformin-associated lactic acidosis (MALA). The severe consequences of an overdose of metformin, as well as the pathophysiologic mechanisms and the clinical consequences of LA in combination with severe hypoglycaemia are described in this report. Early recognition and swift clinical intervention is of paramount importance in case of auto-intoxication with metformin to prevent a lethal outcome.
\end{abstract}

Keywords

Metformin, Lactic Acidosis, Auto-Intoxication

\section{Introduction}

Metformin is the most commonly prescribed oral antihyperglycemic agent in the treatment of type 2 diabetes (DM2) [1]. A potentially life-threatening complication is the occurrence of LA, metformin-associated lactic acidosis (MALA). The incidence is estimated to be 0.03 cases/1000 patient-years [1]. It is generally associated

\footnotetext{
${ }^{\bar{*}}$ Corresponding author.
}

How to cite this paper: Agterhuis, D., Freling, H., Henstra, M. and Veneman, T.F. (2015) Metformin Intoxication: A Case of a Combination of Severe Hypoglycaemia and Severe Lactic Acidosis (LA). Journal of Diabetes Mellitus, 5, 111-114. 
with dehydration, particularly when renal clearance is decreased. If left untreated, its prognosis is poor and mortality rates are high [1].

Since metformin does not stimulate insulin secretion, hypoglycaemia does seldom occur, and is usually ascribed to concurrent use of other antihyperglycemic drugs [2].

We report a case of auto-intoxication with metformin, resulting in a combination of severe LA and severe hypoglycemia of $<0.1 \mathrm{mmol} / \mathrm{l}$.

The purpose is to show the danger of an overdose of the widely used drug metformin, a combination of LA and hypoglycaemia, and the importance of swift recognition and therapeutic intervention.

\section{Case}

A 40-year-old patient was presented to our Emergency Department (ED) after being found unconscious with a Glasgow Coma Scale of E1M1V1. His medical history consisted of DM2 and schizophrenia, treated with metformin, citalopram and aripiprazole, respectively. The time of delay until presentation was unclear but he had been seen in good health 16 hours earlier. Because of the low EMV score the paramedics intubated him, during which he vomited. The stomach contents contained approximately ten pills, consistent with his morning medication.

On arrival at the ED, breathing sounds were normal, oxygensaturation was $100 \%$, blood-pressure $78 / 50$ mmHg, pulse $100 \mathrm{bpm}$, extremities were cold, pupils were symmetrical and not reactive to light, and the rectal temperature was 29.9 Celsius.

Laboratory examination showed a plasma glucoseconcentration $<0.1 \mathrm{mmol} / \mathrm{L}$, CRP $5 \mathrm{mg} / \mathrm{L}$, leukocyte count $32.0 \times 10^{9}$, a severe metabolic acidosis ( $\mathrm{pH} 6.92, \mathrm{pCO}_{2} 6.7 \mathrm{Kpa}$, bicarbonate $10 \mathrm{mmol} / \mathrm{l}, \mathrm{pO}_{2} 10.8$ ), aniongap 34, plasma lactateconcentration (PLC) $18.8 \mathrm{mmol} / \mathrm{L}$ and ethanol $<0.1 \mathrm{~g} / \mathrm{L}$.

A CT-cerebrum and upper spine, performed by the emergency doctor, excluded head and neck trauma.

Urine toxicology screen was negative.

The differential diagnosis included acute metformin intoxication with severe LA and hypoglycemia. The patient was treated with volumesuppletion and glucose-infusion and was admitted to the intensive care unit where hemodialysis was initiated for removal of metformin and lactate. During the following two days hemodialysis was alternated by continuous venovenous hemofiltration $(\mathrm{CVVH})$. However, the PLC initially increased to a maximum of $23.8 \mathrm{mmol} / \mathrm{l}$ whereas the plasma glucoseconcentration showed a tendency towards hypoglycemia. Noradrenaline was needed for hemodynamic support and the patient developed acute renal failure and a severe aspiration pneumonia. A whole body CT was performed to exclude a lymphoreticular malignancy as a cause of hypoglycemia.

After 2 days the PLC finally decreased, the plasma glucoseconcentrations normalized and noradrenaline administration could be tempered. The plasma metformin concentration appeared to be extremely high: $72 \mathrm{mg} / \mathrm{l}$ (therapeutic range 1 - $2 \mathrm{mg} / \mathrm{l}$ ), whereas plasma-insulin and C-peptide-concentrations were normal. After tempering supportive therapy the patient was extubated and discharged to the Internal ward after 9 days. Three days later the patient could be discharged from the hospital in good clinical condition.

\section{Discussion}

If correctly used metformin is a safe drug [1]. Pooled data in a Cochrane review (2010) show no cases of fatal or nonfatal LA in 70,490 patient-years of metformin use nor any changes in PLC [1]. Nevertheless, LA related to metformin use may indeed occur, mostly among patients with renal insufficiency. Bailery et al. descibe 0.03 cases of LA per 1000 patient-years [1]. Primary risk factors for the development of MALA include renal impairment or concomitant illness leading to hypoxemia or renal failure [3].

LA is defined by a blood $\mathrm{pH}<7.35$ and PLC $>5.0 \mathrm{mmol} / \mathrm{l}$. According to the Cohen and Woods classification of 1979 there are 2 types of LA, based on the presence (type A) or absence (type B) of adequate tissue perfusion or oxygenation. MALA is a type B2 LA [1] [3].

The pathogenesis of MALA in metformin overdose is complex and not completely understood [1] [3]. Metformin increases lactateproduction by the intestinal mucosa and suppresses pyruvatecarboxylase, which impairs lactate clearance. Recent research speculates on the role of genes coding for metformin and lactate transporters, which may explain why some metformin users are more sensitive to develop hyperlactacidemia [2].

LA is increasingly observed in patients with overdosing, although the exact incidence remains unknown [2] [4] 
[5]. According to poison control centers LA is seen in 3.5\% of metformin users, and only $16 \%$ of these exposures are due to intentional mono-overdoses with metformin [4]. In most cases serum metformin concentrations are not mentioned [4] [5]. In our case we do not only describe the plasma metformin concentration and plasma lactate at presentation, we also measured concentrations after dialysis, providing valuable information for future research.

Metformin can be cleared easily with dialysis, which is the treatment of choice, since it does not bind to protein. In our patient however, LA initially worsened despite haemodialysis. Presumably, the lactate production was higher than the clearance with haemodialysis, possibly due to a co-existing type A LA due to hypotension. In addition, glucose-infusion may have contributed to the initial worsening of lactatemia since increased metabolism of intracellular glucose may lead to an increase of lactate production [2] [4]. There is evidence that in severe cases with high plasma metformin concentrations, the time of dialysis must be prolonged to ensure clearance of lactate from the intracellular space [5] [6].

The prognosis of LA is poor. However, if LA is caused by metformin in the absence of underlying conditions such as hepatic failure, survival rates are higher [1] [3]. Likewise, higher metformin levels are associated with a better prognosis and therefore the degree of metformin accumulation may even exert a protective effect [3] [7]. The presence of hypotension, acidosis, hyperglycaemia and coma are associated with a poor outcome [7]. Our patient met all these criteria but one (hyperglycemia), but nevertheless survived, possibly due to the fact that LA was caused by mono-metformin intoxication with high plasma metformin concentrations.

Hypoglycemia as low as $<0.1 \mathrm{mmol} / \mathrm{l}$ has not been described previously in metformin users. Metforminassociated hypoglycemia has been reported previously, both in therapeutic dosing as well as in overdosing [5] [8]. The lowest concentration found in the literature is $0.67 \mathrm{mmol} / \mathrm{l}$ in a patient who did not use any other antihyperglycemic agents [6]. In many of the reported cases, sulfonylurea were used concomitantly. Hypoglycemia in metformin monotherapy is generally a consequence of a combination with poor food intake, strenuous exercise, co-ingestion of other anithyperglycemic drugs or comorbidity [5] [9]. Furthermore, some drugs have been associated to interact with metformin, causing hypoglycemia (e.g. rifampicin) [5]. Our patient had a normal nutritional status, no access to sulfonylurea or insulin, no relevant comorbidity and no evidence of co-ingestion of any other medication. The severity of hypoglycemia presented in this case may be explained by the fact that the oral absorption, hepatic uptake, and renal excretion of metformin are largely mediated by organic cation transporters (OCTs). Differences in the level of expression of these transporters in the liver may contribute to a variation in hepatic uptake and clinical effects [2].

The elevated osmolgap at presentation formed a diagnostic dilemma as it is frequently caused by exposure to toxic alcohols [10]. The serum ethanol level in our patient was $<0.1 \mathrm{~g} / \mathrm{L}$ and there was no evidence of ingestion of products containing toxic alcohols. The elevated osmolgap may be explained by extreme hypotension [10] but is has also been previously described in metformin intoxications [4].

\section{Conclusion}

In conclusion, despite the fact that a combination of severe hypoglycemia and severe LA, which are both potentially life-threatening events, was present, our patient survived due to swift diagnosis and therapeutic intervention.

\section{Conflict of Interest}

There is no conflict of interest. No funding was obtained for this report.

\section{References}

[1] Salpeter, S.R., Greyber, E., Pasternak, G.A. and Salpeter, E.E., Editorial Group: Cochrane Metabolic and Endocrine Disorders Group (2010) Risk of Fatal and Nonfatal Lactic Acidosis with Metformin Use in Type 2 Diabetes Mellitus. The Cochrane Database of Systematic Reviews, No. 2, CD002967.

[2] Lalau, J.D., Azzoug, M.L., Kajbaf, F., Briet, C. and Desailloud, R. (2014) Metformin Accumulation without Hyperlactataemia and Metformin-Induced Hyperlactataemia without Metformin Accumulation. Diabetes \& Metabolism, 40, 220-223. http://dx.doi.org/10.1016/j.diabet.2013.12.003

[3] Lalau, J.D. (2010) Lactic Acidosis Induced by Metformin: Incidence, Management and Prevention. Drug Safety, 33, 727-740. http://dx.doi.org/10.2165/11536790-000000000-00000 
[4] Wills, B.K., Bryant, S.M., Buckley, P. and Seo, B. (2010) Can Acute Overdose of Metformin Lead to Lactic Acidosis? The American Journal of Emergency Medicine, 28, 875-861.

[5] Al-Abri, S.A., Hayashi, S., Thoren, K.L. and Olson, C. (2013) Metformin Overdose-Induced Hypoglycemia in the Absence of Other Antidiabetic Drugs. Clinical Toxicology, 51, 444-447. http://dx.doi.org/10.3109/15563650.2013.784774

[6] Avc1, D., Çetinkaya, A., Karahan, S., Oğuzhan, N., Karagöz, H., Başak, M. and Erden, A. (2013) Suicide Commitment with Metformin: Our Experience with Five Cases. Renal Failure, 35, 863-865. http://dx.doi.org/10.3109/0886022X.2013.801299

[7] Spiller, H.A. and Quadrani, D.A. (2004) Toxic Effects from Metformin Exposure. The Annals of Pharmacotherapy, 38, 776-780.

[8] Nathan, D.M., Buse, J.B., Davidson, M.B., Ferrannini, E., Holman, R.R., Sherwin, R. and Zinman, B. (2009) Medical Management of Hyperglycemia in Type 2 Diabetes: A Consensus Algorithm for the Initiation and Adjustment of Therapy: A Consensus Statement of the American Diabetes Association and the European Association for the Study of Diabetes. Diabetes Care, 32, 193-203. http://dx.doi.org/10.2337/dc08-9025

[9] Bodmer, M., Meier, C., Krähenbühl, S., Jick, S.S. and Meier, C.R. (2008) Metformin, Sulfonylureas, or Other Antidiabetes Drugs and the Risk of Lactic Acidosis or Hypoglycemia: A Nested Case-Control Analysis. Diabetes Care, 31, 2086-2091.

[10] Mycyk, M.B. and Aks, S.E. (2003) A Visual Schematic for Clarifying the Temporal Relationship between the Anion and Osmol Gaps in Toxic Alcohol Poisoning. The American Journal of Emergency Medicine, 21, 333-335. 